\title{
A Case Report of Scapulothoracic Dissociation and Literature Review
}

\author{
Fengning Tang, MD, Yong Long, MD, Changluo Li, MD, Ning Ding, MD \\ Department of Emergency Medicine, The Affiliated Changsha Central Hospital, Hengyang Medical School, University of South China
}

\section{ABSTRACT}

Background: Scapulothoracic dissociation (STD) is a rare, life-threatening injury, usually resulting from high energy and leading to poor clinical outcomes and high incidence of disability. This case and a review of the literature aimed to highlight the early diagnosis and management of STD.

Case presentation: A 61-year-old man was injured in a motorcycle accident, when he took a sudden turn and fell from his motorcycle. He was admitted to the emergency department of Changsha Central Hospital. Based on the computed tomography (CT) and digital subtraction angiogram (DSA) findings, the patient was diagnosed as follows: right scapulothoracic dislocation syndrome, right subclavian artery rupture, right subclavian vein rupture, right clavicular fracture, right multiple rib fractures, and right lung contusion and laceration. An expanded polytetrafluoroethylene interposition graft was implemented in the right subclavian artery to re-establish continuity of the subclavian artery. Surgery was performed to repair thoracic deformity, and active rehabilitation therapy also was implemented after surgery. The patient was discharged from the hospital and recovered well with grade 4 muscle strength in his right arm.

Conclusions: Physicians should be vigilant for STD in all patients with shoulder girdle injury. DSA could be recommended for patients with unstable hemodynamics. Early diagnostic image tests and individualized management should be implemented, according to the patient's condition.

\section{INTRODUCTION}

Scapulothoracic dissociation (STD) is a rare, life-threatening injury, usually resulting from high energy and leading to poor clinical outcomes and high incidence of disability [Kani 2019]. STD was described as a complete disruption of the scapulothoracic articulation, which usually presented that the scapular band was separated from the attachment around the scapula, shoulder joint, clavicle and chest wall [Heng 2016].

Received fuly 7, 2021; accepted November 2, 2021.

Correspondence: Ning Ding, MD, Department of Emergency Medicine, The Affiliated Changsha Central Hospital, Hengyang Medical School, University of South China, NO.161 Shaoshan South Road, Changsha, Hunan, 410004, China, Telephone +86 731-8566-7935 (e-mail: doctordingning@csu.edu.cn/ doctordingning@sina.com).
Symptoms and clinical physical examination mainly included a spectrum of musculoskeletal, vascular, and neurologic abnormalities [Choo 2017].

STD was confirmed, based on the medical history as well as clinical and radiographic findings. Diagnosis of STD could be delayed in some patients with multiple traumas. In the present case, a 61-year-old man with STD was diagnosed early and recovered well after surgery. This case and a review of the literature aimed to highlight the early diagnosis and management of STD. The study was approved by the institutional review board of Changsha Central Hospital. Informed consent was obtained from the patient in this study.

\section{CASE PRESENTATION}

A 61-year-old man was injured in a motorcycle accident, when he took a sudden turn and fell from his motorcycle. He was admitted to the emergency department of Changsha Central Hospital at 21:05 on June 25, 2020. On admission, the man was conscious and cooperative with $36.9^{\circ} \mathrm{C}$ body temperature, 115 beats per minute pulse rate, 18 per minute respiratory rate, and $85 / 55 \mathrm{mmHg}$ blood pressure. Physical examination revealed clear heart sound, no murmur in the heart, and no crackles in the lungs. Massive swelling and tenderness on his right neck shoulder, right upper arm, and right upper lateral chest wall were detected. Skin bruise and ecchymosis was detected in his right shoulder. The right upper limb could not be moved, and the pulse of the right radial artery was weakened. Decreased sensation of his right arm was not detected. Lab findings showed a white blood cell count of $10,226 /$ cubic $\mathrm{mm}^{3}$, hemoglobin concentration of $8 \mathrm{~g} /$ $\mathrm{dl}$, platelet count of $127,000 /$ cubic $\mathrm{mm}^{3}$, and an international normalized ratio (INR) of 0.9. No abnormality in liver and kidney function test and coagulation test was detected. The patient reported no special medical history.

With active fluid resuscitation and $4 \mathrm{u}$ blood transfusion, chest computed tomography (CT) was performed and imaging findings showed a fractured right clavicle and fractured ribs (the second to the sixth ribs) with extensive right chest and upper extremity soft tissue swelling. Right hemothorax was also detected on CT. Distances from the spinous process to the inner edge of the scapula on each side were significantly different $(9.1 \mathrm{~cm}$ right vs. $5.1 \mathrm{~cm}$ left). (Figure 1)

A digital subtraction angiogram (DSA) immediately was implemented after the chest CT, and the results revealed that contrast agent was leaking outside the right subclavian and right subclavian artery rupture was confirmed. Spasmed right subclavian vein also was seen in DSA. (Figure 2) We 
prescribed 50mg of meperidine hydrochloride for analgesia. An expanded polytetrafluoroethylene interposition graft was implemented simultaneously in the right subclavian artery to re-establish continuity of the subclavian artery. DSA demonstrated blood flow of the right subclavian artery recovered and no contrast agent overflow. (Figure 3 )

Based on the CT and DSA findings, the patient was diagnosed as follows: right scapulothoracic dislocation syndrome, right subclavian artery rupture, right subclavian vein rupture, right clavicular fracture, right multiple rib fractures, and right lung contusion and laceration. Surgery, including fixation of ruptured clavicle and ribs, was performed for repairing thoracic deformity and active rehabilitation therapy also was implemented after surgery. On July 27 , he was discharged from the hospital and recovered well with grade 4 muscle strength in his right arm.

\section{DISCUSSION AND CONCLUSION}

STD usually occurs due to a high-energy distraction force. With the distraction force increasing, the severity of injury gets worse. A retrospective research with 25 STD patients indicated that more than half occurred as a result of a motorcycle accident [Zelle 2004], as well as in our case. Although most patients were adults, a few immature patients with STD also recently have been reported [Lovejoy 2009].

Asymmetric swelling of the injured shoulder, resulting from hematoma formation and edema, is usually detected in STD. A comprehensive physical examination, including neurologic examination, should be conducted. Diagnostic testing for detecting the severity of injury and evaluating the necessity for surgical intervention also should be performed at the early stage [Althausen 2003]. Vascular injury, which was associated with pulselessness, pallor or coolness of the injured extremity, was common in STD [Knobloch 2006]; this was reported in $61.5 \%$ patients with STD and injured subclavian vessels recently accounted for over 50\% [Zelle 2004]. Vascular injuries, following scapulothoracic dissociation, were associated with long-term clinical outcome. Early CT angiography was recommended for patients with suspected STD [Merchant 2012]. It proved to be an effective method for providing diagnostic information and helped physicians make early decisions. Once vascular injury was identified, surgery including autogenous vein and synthetic graft implantation or endovascular repair should be implemented [Desai 2014]. Recently, endovascular technique has been utilized in more trauma patients, due to its advantages, which could avoid surgical dissection in a traumatized area and significantly decrease the risk of injury to adjoined structures [Jordan 2019]. Research with 1143 trauma patients from 22 institutions in America revealed that endovascular therapy was highly applied among severely injured blunt trauma patients; this was associated with lower incidence of complications and better clinical prognosis [Faulconer 2018]. Another retrospective study demonstrated that endovascular technique could be the first-line treatment for blunt axillo-subclavian arterial injuries in emergent agencies [Matsagkas 2016].

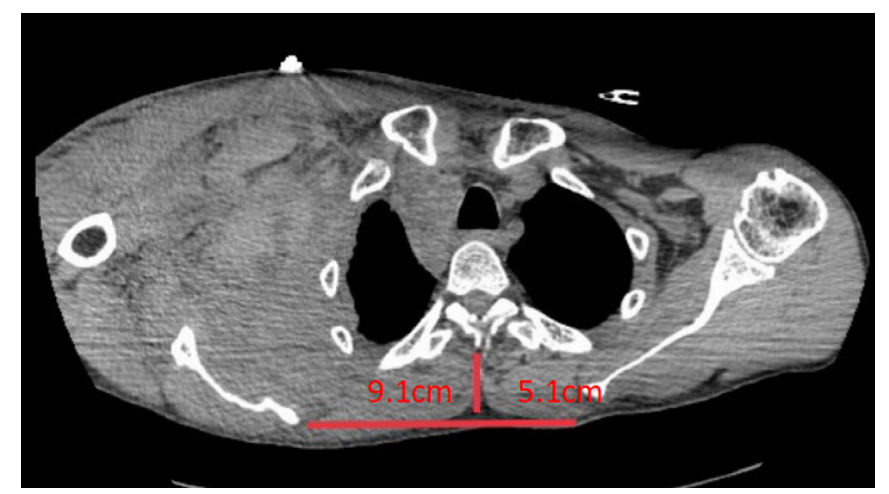

Figure 1. Chest CT demonstrated extensive right chest and upper extremity soft tissue swelled significantly. Comparison between distances from the spinous process to the inner edge of the scapula on each side. Right $(9.1 \mathrm{~cm})$ vs. Left $(5.1 \mathrm{~cm})$.
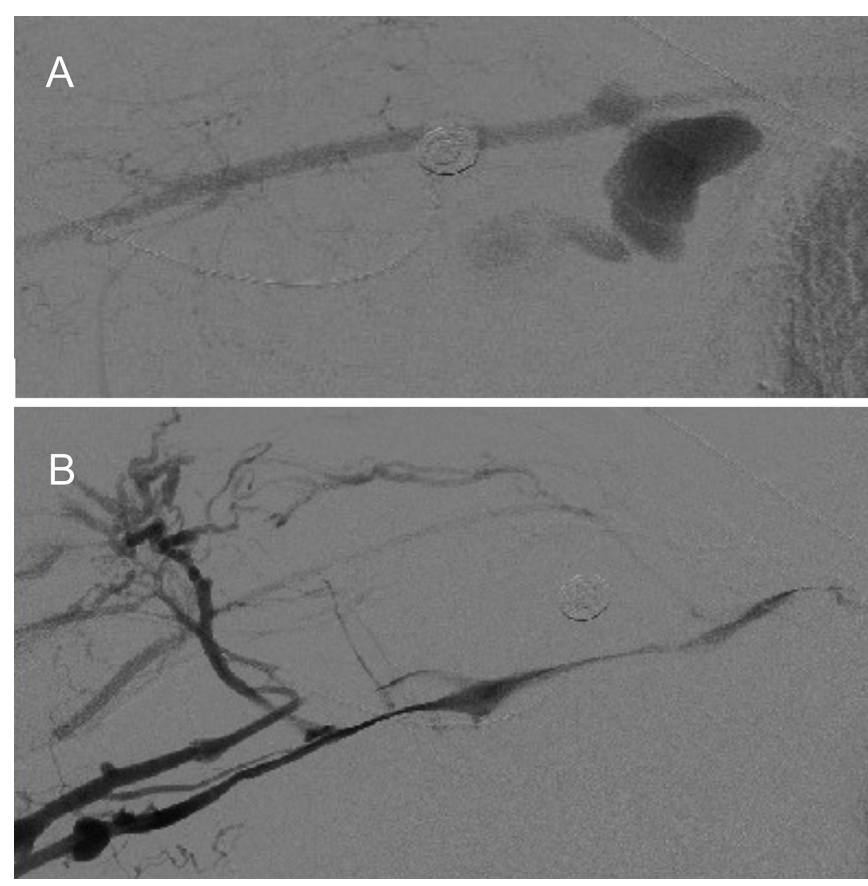

Figure 2. DSA revealed the and right subclavian artery rupture $(A)$ and right subclavian vein spasm (B). DSA, digital subtraction angiogram

In our case, the pulse of right radial artery of the patient was weakened, which apparently indicated the subclavian artery injury. CT was performed at the time of admission, demonstrating significant STD. Eventually, subclavian vascular injuries were confirmed by DSA, and endovascular repair of the subclavian artery was performed.

Brachial plexus injuries may occur in some STD patients with severe trauma, while CT myelography and magnetic resonance image (MRI) are capable of confirming the anatomic level of injury [Rankine 2004]. Recent research illuminated that compared with CT or other imaging, MRI could be better for diagnosing spinal-cord injury and nerve-root avulsion with better accuracy, especially in patients with a 


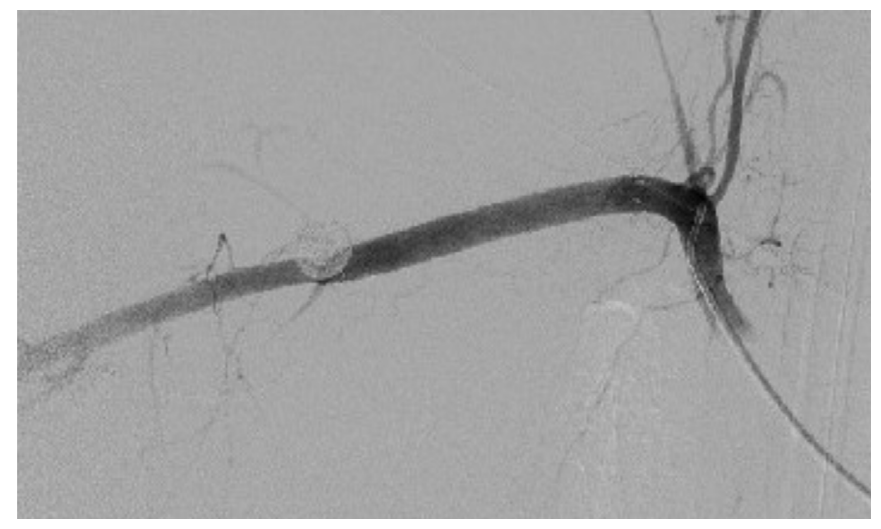

Figure 3. DSA demonstrated that blood flow of the right subclavian artery recovered and no contrast agent overflow. DSA, digital subtraction angiogram

cooperative and hemodynamically stable condition [Lee 2007]. In our case, no symptoms of brachial plexus injuries were detected, so the MRI was not performed.

Recently, more and more patients with STD have been confirmed due to some less severe injuries, which lead to non-typical symptoms and image findings, such as incomplete vascular and neurologic injuries. In our case, CT performed on admission showed significant STD, and vascular injuries immediately were ascertained by DSA. The patient recovered well finally after surgery.

Physicians should be vigilant for STD in all patients with shoulder girdle injury. DSA could be recommended for patients with unstable hemodynamics. Early diagnostic image tests and individualized managements should be implemented, according to the patient's condition.

\section{REFERENCES}

Althausen PL, Lee MA, Finkemeier CG. 2003. Scapulothoracic dissociation: diagnosis and treatment. Clin Orthop Relat Res. (416):237-44.
Choo AM, Schottel PC, Burgess AR. 2017. Scapulothoracic Dissociation. Journal of the American Academy of Orthopaedic Surgeons. 25(5):339-47.

Desai S, DuBose J, Parham C, Charlton-Ouw K, Valdes J, Estrera A, et al. 2014. Outcomes after endovascular repair of arterial trauma. Journal of vascular surgery. 60(5):1309-14

Faulconer ER, Branco BC, Loja MN, Grayson K, Sampson J, Fabian TC, et al. 2018. Use of open and endovascular surgical techniques to manage vascular injuries in the trauma setting: A review of the American Association for the Surgery of Trauma PROspective Observational Vascular Injury Trial registry. J Trauma Acute Care Surg. 84(3):411-7.

Heng K. 2016. "Floating shoulder" injuries. Int J Emerg Med. 9(1):13.

Jordan R, Obmann M, Song B, Nikam S, Mariner D, Toy F, et al. 2019. Hybrid approach to complex vascular injury secondary to blast induced scapulothoracic dissociation. Trauma Case Reports. 23.

Kani KK, Chew FS. 2019. Scapulothoracic dissociation. Br J Radiol. 92(1101):20190090

Knobloch K, von Falck C, Teebken O, Krettek C. 2006. Scapulothoracic dissociation with subclavian artery dissection following a severe motorbike accident. Eur J Cardiothorac Surg. 30(4):671.

Lee GK, Suh KJ, Choi JA, Oh HY. 2007. A case of scapulothoracic dissociation with brachial plexus injury: magnetic resonance imaging findings. Acta Radiol. 48(9):1020-3.

Lovejoy J, Ganey TM, Ogden JA. 2009. Scapulothoracic dissociation secondary to major shoulder trauma. J Pediatr Orthop B. 18(3):131-4.

Matsagkas M, Kouvelos G, Peroulis M, Xanthopoulos D, Bouris V, Arnaoutoglou E. 2016. Endovascular repair of blunt axillo-subclavian arterial injuries as the first line treatment. Injury. 47(5):1051-6.

Merchant N, Scalea T, Stein D. 2012. Can CT angiography replace conventional bi-planar angiography in the management of severe scapulothoracic dissociation injuries? The American surgeon. 78(8):875-82.

Rankine J. 2004. Adult traumatic brachial plexus injury. Clinical radiology. 59(9):767-74.

Zelle B, Pape H, Gerich T, Garapati R, Ceylan B, Krettek C. 2004. Functional outcome following scapulothoracic dissociation. The Journal of bone and joint surgery. American volume. 86(1):2-8. 